Original Research Paper

\title{
An Expansion Iterative Technique for Handling Fractional Differential Equations Using Fractional Power Series Scheme
}

\author{
${ }^{1}$ Radwan Abu-Gdairi, ${ }^{2}$ Mohammed Al-Smadi and ${ }^{3}$ Ghaleb Gumah \\ ${ }^{I}$ Department of Mathematics, Faculty of Science and Information Technology, \\ Zarqa Private University, Zarqa 13110, Jordan \\ ${ }^{2}$ Applied Science Department, Ajloun College, Al-Balqa Applied University, Ajloun 26816, Jordan \\ ${ }^{3}$ Applied Science Department, Faculty of Engineering Technology, \\ Al-Balqa Applied University, Amman 11942, Jordan
}

Article history

Received: 16-07-2015

Revised: 20-07-2015

Accepted: 29-08-2015

Corresponding Author: Mohammed Al-Smadi Applied Science Department, Ajloun College, Al-Balqa Applied University, Ajloun 26816, Jordan

E-mail: mhm.smadi@yahoo.com

\begin{abstract}
In this study, we present a new analytical numerical technique for solving a class of time Fractional Differential Equations (FDEs) with variable coefficients based on the generalized Taylor series formula in the Caputo sense. This method provided the solution in the form of a rapidly convergent power series under a multiple fractional differentiability with easily computable components. An efficacious experiment is given to guarantee the procedure, to illustrate the theoretical statements of the present technique and to show its potentiality, generality and superiority for solving wide range of FDEs. The results reveal that the method is easy to implement, very effective, fully compatible with the complexity of such problems, straightforward and simple.
\end{abstract}

Keywords: Fractional Differential Equation, Residual Power Series Method, Approximate Solution, Series Expansion Representation

\section{Introduction}

The FDEs are increasingly used to model many problems in mathematical physics, including electromagnetic, fluid flow, diffusion, quantum mechanics, damping laws, viscoelasticity and other applications (Oldham and Spanier, 1974; Miller and Ross, 1993; Young, 1995; Mainardi, 1997; Luchko and Gorenflo, 1998; He, 1999; Podlubny, 1999; Caputo, 1967; Shawagfeh, 1999; Shawagfeh, 2002; Momani and Shawagfeh, 2002). Exact analytical solutions of FDEs are sometimes too complicated to be solved by conventional techniques due to the computational complexities of fractional calculus involving them. The solvability of linear FDEs has been devoted on terms of special constraint condition; whereas in general, there exists no method dealing with the existence of solutions of nonlinear FDEs. So it required to derive an efficient approximate solution. It is little wonder that with the development of fast, efficient digital computers, the role of numerical methods in mathematics, physics and engineering problem solving has increased dramatically in recent years. As a result, the solution of such equations can be approximated with a large variety of analytical numerical methods. Among these, homotopy perturbation method (Momani and Odibat, 2007; Momani and Odibat, 2007; Odibat and Momani, 2008), variational iteration method (Odibat and Momani,
2006; Abbasbandy, 2007; Molliq et al., 2009), homotopy analysis method (Song and Zhang, 2007; Hashim et al., 2009; El-Ajou et al., 2010; Arqup and El-Ajou, 2013), transform methods (Arikoğlu and Özkol, 2007; Oturanç et al., 2008; Ertürk and Momani, 2008; Momani et al., 2014) and other methods (Schneider, 1996; Arqub et al., 2012; Al-Smadi et al., 2013; Arqub et al., 2013; Arqub et al., 2015).

Theory of fractional calculus provides an excellent instrument for description of dynamical behaviors, memory and hereditary properties to model physical processes and systems. Techniques of fractional calculus have been employed at the modeling of many different phenomena in applied science. The most important advantage for using these equations is a nonlocal property. It should be noted that the integer order differential or integral operators are local, while on the other hand, the fractional order differential or integral operators are nonlocal. This means that the next status of a model depends not only upon its current status but also upon all of its historical situations. This generalization showed many new fundamentals which exist in the fractional order domain and disappear in the integer order case, helped to deeper understanding of abovementioned natural events, reduced computational complexity and explained even more complex structures. Indeed, series expansions are very important aids in 
numerical calculations, especially for quick estimates made in hand calculation. Solutions to FDEs can often be expressed in terms of series expansions. The Fractional Power Series (FPS) method is an approximate as well as analytical method for solving many types of ODEs and PDEs of fractional order. The FPS methodology provides the solution in terms of convergent power series under a multiple fractional order with easily computable components. It is effective and easy to construct power series solution for strongly linear and nonlinear equations without linearization, perturbation, or discretization (Arqub, 2013; Al-Smadi, 2013; Moaddy et al., 2015; El-Ajou et al., 2015; Komashynska et al., 2015). Different from the classical power series method, the FPS method does not need to compare the coefficients of the corresponding terms and a recursion relation is not required. The Taylor series method is computationally expensive for large orders and suited for the linear problems, while on the other hand, the proposed method is an alternative procedure for obtaining analytic Taylor series solution of FDEs and computes the coefficients of the power series by a chain of algebraic equations of one or more variables. By concept of residual error, we get a series solution in practice as well a truncated series solution. This series solution does not exhibit the real behaviors of the problem but gives a good approximation to the true solution in the given region.

The objective of the present work is to extend the application of the FPS method to derive fractional series solutions for time fractional wave-like differential equation with variable coefficients based on the generalized Taylor series formula in the Caputo sense. The outline of this paper is organized as follows. In the next section, we utilize some necessary definitions and results from fractional calculus theory. In section 3, basic idea of the FPS method is presented in order to construct and predict solutions of the FDEs. Description of the FPS method is given in section 4. In section 5, application and formulation of the solution for fractional wave-like differential equation are performed in order to illustrate the capability and simplicity of proposed method. The conclusions are given in last section.

\section{Overview of Fractional Calculus Theory}

The material in this section is basic in certain sense. For the reader's convenience, we summarize some concepts, definitions and basic results from fractional calculus in the sequel, which are useful in the following discussion. These definitions and concepts can be found in (Oldham and Spanier, 1974; Miller and Ross, 1993; Young, 1995; Mainardi, 1997; Luchko and Gorenflo, 1998) and references therein. Throughout this analysis, the set of real numbers and the set of integer numbers are denoted by $\mathrm{R}, \mathrm{N}$, and respectively, whilst the Gamma function is denoted by $\Gamma$ Firstly, we shall introduce a modified fractional differential operator proposed by Caputo in his work on the theory of viscoelasticity (Caputo, 1967).

Definition 1. A real function $f(x), x>0$, is said to be in the space $C_{\mu}, \mu \in \mathbb{R}$, if there exists a real number $p>$ $\mu$ such that $f(x)=x^{\mathrm{p}} f_{1}(\mathrm{x})$, where $f_{1}(x) \in \mathrm{C}[0, \propto)$ and it is said to be in the space $C_{\mu}^{n}$ if $f^{(n)}(x) \in C_{\mu}, n \in \mathbb{N}$.

Definition 2. The Riemann-Liouville integral operator of order $\mathrm{a} \geq 0$ of a function $f(x) \in \mathrm{C}_{\mu}, \mu \geq-1$ is defined as:

$$
J_{s}^{a} f(x)=\left\{\begin{array}{ccr}
\frac{1}{\Gamma(a)} \int_{s}^{x}(x-t)^{a-1} f(t) d t, & x>t>s \geq 0, a>0 \\
f(x), & a & =0
\end{array}\right.
$$

Consequently, the operator $J_{s}^{a}$ has the following properties: For $f(x) \in \mathrm{C}_{\mu}, \mu \geq-1, \mathrm{a}, \beta \geq 0, \mathrm{c} \in \mathbb{R}$ and $\gamma>$ 1, we have $J_{s}^{a} J_{s}^{\beta} f(x)=J_{s}^{a+\beta} f(x)=J_{s}^{\beta} J_{s}^{a} f(x), J_{s}^{a} C=\frac{C}{\Gamma(a+1)}(x-S)^{a}$ and $J_{s}^{a}(x-S)^{\gamma}=\frac{\Gamma(\gamma+1)}{\Gamma(a+\gamma+1)}(x-S)^{a+\gamma}$.

Definition 3. The Riemann-Liouville integral operator of order a $\geq 0$ of a function $f(x) \in C_{-1}^{n}, n \in \mathbb{N}$ is defined as:

$\hat{D}_{s}^{a} f(x)=\left\{\begin{array}{lrl}\frac{d^{n}}{d x^{n}} J^{n-a} f(x), & n-1<a<n \\ \frac{d^{n}}{d x^{n}} f(x), & a=n\end{array}\right.$

Next, we will introduce a modified fractional differential operator $D_{s}^{a}$.

Definition 4. The Caputo fractional derivative operator of order a $>0$ of a function $f(x) \in C_{-1}^{n}, n \in \mathbb{N}$ is defined as:

$D_{s}^{a} f(x)=\left\{\begin{array}{cc}J_{s}^{n-a} f^{(n)}(x), & x-s \geq 0, n-1<a<n \\ \frac{d^{n}}{d x^{n}} f(x), & a=n\end{array}\right.$

For some certain properties of the operator $D_{s}^{a}$ we have $\quad D_{s}^{a}(x-S)^{\gamma}=\frac{\Gamma(\gamma+1)}{\Gamma(a+\gamma+1)}(x-S)^{\gamma-a}$ and $\quad D_{s}^{a} c=0$, where $\gamma>-1, a \geq 0$ and $c \in \mathbb{R}$.

Theorem 1. If $n-1<a \leq n, f(x) \in C_{\mu}^{n}, n \in \mathbb{N}$ and $\mu \geq-1$, then $D_{s}^{a} J_{s}^{a} f(x)=f(x)$ and

$J_{s}^{a} D_{s}^{a} f(x)=f(x)-\sum_{k=0}^{n-1} f^{(k)}\left(s^{+}\right) \frac{(x-s)^{k}}{k !}$, where $x>\mathrm{s} \geq 0$. 


\section{Fractional Power Series Representation}

In this section, we will introduce a general form of generalized Taylor's formula that containing the Caputo's definition for fractional derivatives together with some theories and results, which we can formulate any function with certain properties in term of its FPS representation.

Definition 5. A power series representation of the form:

$$
\sum_{n=0}^{\infty} c_{n}\left(t-t_{0}\right)^{n a}=c_{0}+c_{1}\left(t-t_{0}\right)^{a}+c_{2}\left(t-t_{0}\right)^{2 a}+\ldots
$$

where, $0 \leq m-1<a \leq m$ and $t \geq t_{0}$ is called a FPS expansion about $\mathrm{t}=\mathrm{t}_{0}$, where $\mathrm{t}$ is a variable and $c_{n}$ 's are constants called the coefficients of the series.

As a special case, when $t_{0}=0$, the series $\sum_{n=0}^{\infty} c_{n} t^{n a}$ is called a fractional Maclaurin series. Notice that in writing out the term corresponding to $n=0$ in Equation (4), we have adopted the convention that $\left(t-t_{0}\right)^{0}=1$ even when $t=t_{0}$. Also, when $t=t_{0}$ each terms of Equation (4) are vanishing for $n \neq 0$ and so. On the other hand, the FPS representation of Equation (4) always converges when $t=t_{0}$.

Theorem 2. The CPS expansion $\sum_{n=0}^{\infty} c_{n} t^{n},-\infty<t<\infty$ has radius of convergence $R$ if and only if the FPS expansion $\sum_{n=0}^{\infty} c_{n} t^{n a}, t \geq 0$ has radius of convergence $R^{1 / a}$.

Proof. If we make change of variable $t=x^{\mathrm{a}}, x \geq 0$, then the CPS expansion $\sum_{n=0}^{\infty} c_{n} t^{n}$ becomes $\sum_{n=0}^{\infty} c_{n} x^{a n}$ which is converges for $0 \leq x^{\mathrm{a}} \leq R$, that is, for $0 \leq x \leq R^{1 / \mathrm{a}}$. So, the FPS $\sum_{n=0}^{\infty} c_{n} x^{a n}$ has radius of convergence $R^{1 / a}$. Conversely, if we make change of variable $\mathrm{t}=x^{1 / a}, x \geq 0$, then the FPS expansion $\sum_{n=0}^{\infty} c_{n} n^{n a}$ becomes $\sum_{n=0}^{\infty} c_{n} x^{n}, x \geq 0$, which is converges for $0 \leq x^{1 / a} \leq R^{1 / a}$, that is, for $0 \leq x \leq R$. Since $\sum_{n=0}^{\infty} c_{n} x^{n}, x \geq 0$ and $\sum_{n=0}^{\infty} c_{n} x^{n},-\propto<x<\propto$ have the same radius of convergence $R=\lim _{n \rightarrow \infty}\left|\frac{c_{n}}{c_{n+1}}\right|$, then the CPS $\sum_{n=0}^{\infty} c_{n} t^{n},-\infty$ $<t<\propto$ has radius of convergence $\mathrm{R}$.

Lemma 1. Suppose that the FPS expansion $\sum_{n=0}^{\infty} c_{n}\left(t-t_{0}\right)^{n a}, 0 \leq m-1<a \leq m t \geq, t_{0}$ has radius of convergence $\mathrm{R}>0$. If the function $f$ defined by $\sum_{n=0}^{\infty} c_{n}\left(t-t_{0}\right)^{n a}$, then for $\mathrm{t}_{0}<t<R$ the following hold:

$$
\begin{aligned}
& D_{t_{0}}^{a} f(t)=\sum_{n=1}^{\infty} c_{n} \frac{\Gamma(n a+1)}{\Gamma((n-1) a+1)}\left(t-t_{0}\right)^{(n-1) a} \\
& J_{t_{0}}^{a} f(t)=\sum_{n=0}^{\infty} c_{n} \frac{\Gamma(n a+1)}{\Gamma((n+1) a+1)}\left(t-t_{0}\right)^{(n+1) a}
\end{aligned}
$$

Proof. From Equation (1) and (3) and certain properties of the operator $D_{s}^{a}$ and $J_{s}^{a}$, one can conclude that:

$$
\begin{aligned}
& D_{t_{0}}^{a} f(t)=\frac{1}{\Gamma(m-a)} \int_{0}^{x}(x-\tau)^{m-a-1} f^{(m)}(\tau) d \tau \\
& =\frac{1}{\Gamma(m-a)} \int_{t_{0}}^{x}(x-\tau)^{m-a-1}\left(\frac{d^{m}}{d \tau^{m}} \sum_{n=0}^{\infty} c_{n}\left(\tau-t_{0}\right)^{n a}\right) d \tau \\
& =\frac{1}{\Gamma(m-a)} \int_{t_{0}}^{x}(x-\tau)^{m-a-1}\left(\sum_{n=0}^{\infty} c_{n} \frac{d^{m}}{d \tau^{m}}\left(\tau-t_{0}\right)^{n a}\right) d \tau \\
& =\sum_{n=1}^{\infty} c_{n} \frac{1}{\Gamma(m-a)} \int_{t_{0}}^{x}(x-\tau)^{m-a-1}\left(\frac{d^{m}}{d \tau^{m}}\left(\tau-t_{0}\right)^{n a}\right) d \tau \\
& =\sum_{n=1}^{\infty} c_{n} D_{t_{0}}^{a}\left(\left(t-t_{0}\right)^{n a}\right)=\sum_{n=1}^{\infty} c_{n} \frac{\Gamma(n a+1)}{\Gamma((n-1) a+1)}\left(t-t_{0}\right)^{(n-1) a}
\end{aligned}
$$

For the other part, by applying the operator $J_{s}^{a}$ and using $J_{s}^{a}(x-s)^{\gamma}=\frac{r(\gamma+1)}{r(a+\gamma+1)}(x-s)^{a+\gamma}$, one can conclude that:

$$
\begin{aligned}
& J_{t_{0}}^{a} f(t)=\frac{1}{\Gamma(a)} \int_{t_{0}}^{x}(x-\tau)^{a-1} f(\tau) d \tau \\
& =\frac{1}{\Gamma(a)} \int_{t_{0}}^{x}(x-\tau)^{a-1}\left(\sum_{n=0}^{\infty} c_{n}\left(\tau-t_{0}\right)^{a n}\right) d \tau \\
& =\sum_{n=0}^{\infty} c_{n}\left(\frac{1}{\Gamma(a)} \int_{t_{0}}^{x}(x-\tau)^{a-1}\left(\left(\tau-t_{0}\right)^{a n} d \tau\right)=\sum_{n=0}^{\infty} c_{n} J_{t_{0}}^{a}\left(\left(t-t_{0}\right)^{a n}\right)\right. \\
& =\sum_{n=0}^{\infty} c_{n} \frac{\Gamma(a n+1)}{\Gamma((n+1) a+1)}\left(t-t_{0}\right)^{(n+1) a}
\end{aligned}
$$

So the proof of the lemma is complete.

Theorem 3. Suppose that $f$ has a FPS representation at $t=t_{0}$ of the form:

$$
f(t)=\sum_{n=0}^{\infty} c_{n}\left(t-t_{0}\right)^{n a}, 0 \leq m-1<a \leq m, t_{0} \leq t<t_{0}+R
$$

If $f(\mathrm{t}) \in \mathrm{C}\left[t_{0}, t_{0}+R\right], \quad D_{t_{0}}^{n a} f(t) \in C\left(t_{0}, t_{0}+R\right) \quad$ and $D_{t_{0}}^{n a} f(t)$ can be differentiated $(m-1)$ - times on $\left(\mathrm{t}_{0}, \mathrm{t}_{0}+\mathrm{R}\right)$ for $n=0,1,2, \ldots$, then the coefficients $c_{n}$ of Equation (7) are given by the formula:

$c n=\frac{D_{t_{0}}^{n a} f\left(t_{0}\right)}{\Gamma(n a+1)}, n=0,1,2, \ldots$,

where, $D_{t_{0}}^{n a}=D_{t_{0}}^{a} \cdot D_{t_{0}}^{a} \ldots \ldots . D_{t_{0}}^{a}$ (n-times).

Proof. Let $f$ be an arbitrary function that can be represented by the FPS expansion of Equation (4). If we put $t=t_{0}$ back into Equation (5), then all terms are vanishing except the first will be $c_{0}=f\left(t_{0}\right)$. 
On the other aspect as well, by using the result of Equation (5), one can obtain:

$$
\begin{aligned}
& D_{t_{0}}^{a} f(t)=c_{1} \Gamma(a+1)+c_{2} \\
& \frac{\Gamma(2 a+1)}{\Gamma(a+1)}\left(t-t_{0}\right)^{a}+c_{3} \frac{\Gamma(3 a+1)}{\Gamma(2 a+1)}\left(t-t_{0}\right)^{2 a}+\ldots, t_{0} \\
& \leq t<t_{0}+R
\end{aligned}
$$

The substitution of $t=t_{0}$ back into Equation (9) leads to $c_{1}=\frac{D_{t_{0}}^{a} f\left(t_{0}\right)}{\Gamma(a+1)}$. Thus, applying the operator $D_{t_{0}}^{a}$ on the series representation of Equation (9), it obtains that:

$$
\begin{aligned}
& D_{t_{0}}^{2 a} f(t)=c_{2} \Gamma(2 a+1) \\
& +c_{3} \frac{\Gamma(3 a+1)}{\Gamma(a+1)}\left(t-t_{0}\right)^{a}+c_{4} \frac{\Gamma(4 a+1)}{\Gamma(2 a+1)}\left(t-t_{0}\right)^{2 a}+\ldots, t_{0} \\
& \leq t<t_{0}+R
\end{aligned}
$$

Again, if we substitute $\mathrm{t}=\mathrm{t}_{0}$ back into Equation (10), then the result is $c_{2}=\frac{D_{t_{0}}^{2 a} f\left(t_{0}\right)}{\Gamma(2 a+1)}$. By using similar fashion if we continue to operate $D_{t_{0}}^{a}$ n-times and then substitute $t=t_{0}$, then the successive pattern for $c_{n}$ will be obtained. Consequently, the general formula of $c_{\mathrm{n}}$ in Equation (7) is given by $c_{2}=\frac{D_{t_{0}}^{n a} f\left(t_{0}\right)}{\Gamma(n a+1)}, n=0,1,2, \ldots$.

So, the proof of the theorem is completely constructed.

It worth here to mention that the substituting of general form $c_{n}=\frac{D_{t_{0}}^{n a} f\left(t_{0}\right)}{\Gamma(n a+1)}, n=0,1,2$ back into the series representation of Equation (7) leads to the following expansion of $f(\mathrm{t})$ about $\mathrm{t}_{0}$ :

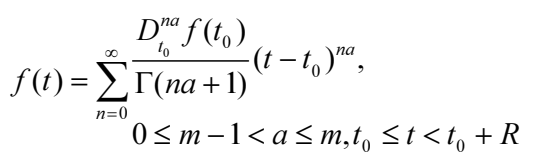

Which is the generalized Taylor's formula derived by Odibat and Shawagfeh (2007) for $0<a \leq 1$. Moreover, in case of $a=m, m \in \mathbb{N}$, the general form of the generalized Taylor's series formula of Equation (11) reduces to the classical Taylor's formula.

Next, we will present the following lemma in order to give a bound for the remainder of error expansion to capture the behavior of the approximating solutions.

Lemma 2. Suppose that $f(t) \in \mathrm{C}\left[t_{0}, t_{0}+R\right]$ and $D_{t_{0}}^{k a} f(t) \in C\left(t_{0}, t_{0}+R\right)$ for $k=0,1,2, \ldots, n+1$, where $0<\mathrm{a} \leq$ 1. Then:

$$
\begin{aligned}
& J_{t_{0}}^{(n+1) a} D_{t_{0}}^{(n+1) a} f(t)=f(t) \\
& -\sum_{k=0}^{n} \frac{D_{t_{0}}^{k a} f\left(t_{0}\right)}{\Gamma(k a+1)}\left(t-t_{0}\right)^{k a}, t_{0} \leq t<t_{0}+R
\end{aligned}
$$

Proof. From the certain properties of the operator $J_{t_{0}}^{a}$, we have:

$$
\begin{aligned}
& J_{t_{0}}^{(n+1) a} D_{t_{0}}^{(n+1) a} f(t)= \\
& =J_{t_{0}}^{n a}\left(\left(J_{t_{0}} D_{t_{0}}\right) D_{t_{0}}^{n a} f(t)\right)=J_{t_{0}}^{n a}\left(D_{t_{0}}^{n a} f(t)-D_{t_{0}}^{n a} f\left(t_{0}\right)\right) \\
& =J_{t_{0}}^{n a} D_{t_{0}}^{n a} f(t)-J_{t_{0}}^{n a}\left(D_{t_{0}}^{n a} f\left(t_{0}\right)\right) \\
& =J_{t_{0}}^{(n-1) a}\left(\left(J_{t_{0}} D_{t_{0}}\right) D_{t_{0}}^{(n-1) a} f(t)\right)-\left(\frac{D_{t_{0}}^{n a} f\left(t_{0}\right)}{\Gamma(n a+1)}\left(t-t_{0}\right)^{n a}\right) \\
& =J_{t_{o}}^{(n-1) a} D_{t_{0}}^{(n-1) a} f(t)-J_{t_{0}}^{(n-1) a}\left(D_{t_{0}}^{(n-1) a} f\left(t_{0}\right)\right)-\left(\frac{D_{t_{0}}^{n a} f\left(t_{0}\right)}{\Gamma(n a+1)}\left(t-t_{0}\right)^{n a}\right) \\
& =J_{t_{0}}^{(n-2) a}\left(\left(J_{t_{0}} D_{t_{0}}\right) D_{t_{0}}^{(n-2) a} f(t)\right)-\left(\frac{D_{t_{0}}^{(n-1) a} f\left(t_{0}\right)}{\Gamma((n-1) a+1)}\left(t-t_{0}\right)^{(n-1) a}\right) \\
& -\left(\frac{D_{t_{0}}^{n a} f\left(t_{0}\right)}{\Gamma(n a+1)}\left(t-t_{0}\right)^{n a}\right) \\
& =J_{t_{0}}^{(n-2) a}\left(D_{t_{0}}^{(n-2) a} f(t)-D_{t_{0}}^{(n-2) a} f\left(t_{0}\right)\right)-\sum_{k=n-1}^{n} \frac{D_{t_{0}}^{k a} f\left(t_{0}\right)}{\Gamma a+1)}\left(t-t_{0}\right)^{k a} \\
& =J_{t_{0}}^{(n-2) a} D_{t_{0}}^{(n-2) a} f(t)-\sum_{k=n-2}^{n} \frac{D_{t_{0}}^{k a} f\left(t_{0}\right)}{\Gamma(k a+1)}\left(t-t_{0}\right)^{k a}=\ldots \\
& =J_{t_{0}}^{a} D_{t_{0}}^{a} f(t)-\sum_{k=1}^{n} \frac{D_{t_{0}}^{k a} f\left(t_{0}\right)}{\Gamma(k a+1)}\left(t-t_{0}\right)^{k a}=f(t)-\sum_{k=0}^{n} \frac{D_{t_{0}}^{k a} f\left(t_{0}\right)}{\Gamma(k a+1)}\left(t-t_{0}\right)^{k a}
\end{aligned}
$$

This completes the proof.

Theorem 4. If $\left|D_{t_{0}}^{(n+1) a} f(t)\right| \leq M$ on $t_{0} \leq t \leq b$, where 0 $<a \leq 1$, then the reminder $R_{n}(t)$ of the Generalized Taylor's series will satisfies the inequality:

$$
\left|R_{n}(t)\right| \leq \frac{M}{\Gamma((n+1) a+1)}\left(t-t_{0}\right)^{(n+1) a}
$$

Proof. Assume that $D_{t_{0}}^{k a} f(t)$ exist for $k=0,1,2, \ldots, n+1$ and $\left|D_{t_{0}}^{(n+1) a} f(t)\right| \leq M$, then from the definition of the reminder $\quad R_{n}(t)=f(t)-\sum_{k=0}^{n} \frac{D_{t_{0}}^{k a} f\left(t_{0}\right)}{\Gamma(k a+1)}\left(t-t_{0}\right)^{k a}$, one can obtain $\quad R_{n}\left(t_{0}\right)=D_{t_{0}}^{a} R_{n}\left(t_{0}\right)=D_{t_{0}}^{2 a} R_{n}\left(t_{0}\right)=\ldots=D_{t_{0}}^{n a} R_{n}\left(t_{0}\right)=0$ and $\quad D_{t_{0}}^{(n+1)} R_{n}(t)=D_{t_{0}}^{(n+1) a} f(t), t_{0} \leq t \leq b . \quad$ Thus, $-M \leq D_{t_{0}}^{(n+1) a} f(t) \leq M$, for each $t_{0} \leq t \leq b$ and hence the operator $J_{t_{0}}^{a}$ should satisfy: 


$$
J_{t_{0}}^{(n+1) a}(-M) \leq J_{t_{0}}^{(n+1) a} D_{t_{0}}^{(n+1) a} f(t) \leq J_{t_{0}}^{(n+1) a}(M)
$$

From the above lemma, we obtain that $J_{t_{0}}^{(n+1) a} D_{t_{0}}^{(n+1) a} f(t)=R_{n}(t)$. By performing the operations in Equation (14), the inequality $-M \frac{\left(t-t_{0}\right)^{(n+1) a}}{\Gamma((n+1) a+1)} \leq R_{n}(t) \leq M \frac{\left(t-t_{0}\right)^{(n+1) a}}{\Gamma((n+1) a+1)} \quad$ must $\quad$ be holds, which is equivalent to $\left|R_{n}(t)\right| \leq \frac{M}{\Gamma((n+1) a+1)}\left(t-t_{0}\right)^{(n+1) a}, t_{0} \leq t \leq b$. So, the proof is complete.

Theorem 5. If a function $f$ has a FPS representation at $\mathrm{t}_{0}$, that is:

$$
f(t)=\sum_{n=0}^{\infty} c_{n}\left(t-t_{0}\right)^{n a}, 0 \leq m-1<a \leq m, t_{0} \leq t<t_{0}+R
$$

where, is the radius of convergence, then $f(\mathrm{t})$ is analytic in the interval $\left(t_{0}, t_{0}+R\right)$.

Proof. Let $g(t)=\sum_{n=0}^{\infty} c_{n} t^{n},|t|<R^{a}$ and $h(t)=\left(t-t_{0}\right)^{a}, t_{0}$ $<t, 0 \leq m-1<a \leq m$. Then $\mathrm{g}(\mathrm{t})$ and $\mathrm{h}(\mathrm{t})$ are analytic functions and thus the composition $(g \circ h)(t)=f(t)$ is analytic in $\left(t_{0}, t_{0}+R\right)$. This completes the proof.

\section{Description of the FPS Method}

In this section, we construct and obtain analytical solutions for FDEs subject to given initial conditions based on the generalized Taylor's formula that involving Caputo fractional derivatives by substituting its fractional PS expansion among its truncated residual function. From the resulting equation a recursion formula for the computation of the coefficients is derived, while the coefficients in the fractional PS expansion can be computed recursively by recurrent fractional differentiation of the truncated residual function. To achieve our goal, let us consider the following form of nonlinear FDEs:

$$
\begin{aligned}
& D_{t_{0}}^{a} u(t)+a_{m} u^{(m)}(t)+a_{m-1} u^{(m-1)}(t)+\ldots+ \\
& a_{1} u^{\prime}(t)+a_{0} u(t)+N\left(u(t), u^{\prime}(t)\right)=g(t)
\end{aligned}
$$

Subject to the initial conditions:

$u^{(i)}\left(t_{0}\right)=b_{i}, i=0,1,2, \ldots, m-1$

where, $t \geq t_{0}, m-1 \leq a \leq m, m \in \mathbb{N}, \mathrm{a}_{\mathrm{i}}$ and $\mathrm{b}_{\mathrm{i}}$ are known real constants, $N(\cdot)$ is a nonlinear operator, $\mathrm{g}(\mathrm{t})$ is known function can be expanded in the general form of the generalized Taylor's formula and $u(t)$ is unknown function to be determined.

To do so, we first illustrate the basic idea of the RPS method for solving the selected equation analytically.
The RPS method consists in expressing the solution of FIVP (16) and (17) as a fractional PS expansion about the initial point $t_{0}$. Consequently, we suppose that the solution $u(t)$ takes the following expansion form:

$u(t)=\sum_{j=0}^{\infty} c_{j} \frac{\left(t-t_{0}\right)^{j a}}{\Gamma(j a+1)}, t \geq t_{0}$

The RPS provides an analytical approximate solution in terms of an infinite FPS. However, to obtain numerical values from this series, the consequent series truncation and the practical procedure are conducted to accomplish this task. In the following step, we will let $\mathrm{u}_{\mathrm{k}}(\mathrm{t})$ o denote the $\mathrm{k}-\mathrm{th}$ truncated series of $u(t)$. That is:

$u_{k}(t)=\sum_{j=0}^{k} c_{j} \frac{\left(t-t_{0}\right)^{j a}}{\Gamma(j a+1)}, t \geq t_{0}$

Since $u(t)$ satisfies the initial conditions (17), so from Equation (18), we obtain $u\left(t_{0}\right)=c_{0}=b_{0}$. On the other hand, from Equation (19) the initial guess approximation of $\mathrm{u}(\mathrm{t})$ is $\mathrm{u}_{0}(\mathrm{t})=\mathrm{c}_{0}=\mathrm{b}_{0}$. As a result, we can reformulate the expansion of Equation (19) in the following form:

$u_{k}(t)=b_{0}+\sum_{j=1}^{k} c_{j} \frac{\left(t-t_{0}\right)^{j a}}{\Gamma(j a+1)}, t \geq t_{0}, k=1,2,3, \ldots$

Prior to applying the fractional RPS technique for finding the values of the coefficients $c_{j}, j=1,2, \ldots, k$, of the series expansion of Equation (20), we define the residual function, Res, for the FDE (16) as follows:

$\operatorname{Res}(t)=D_{t_{0}}^{a} u(t)-f\left(t, u(t), u^{\prime}(t), u^{\prime \prime}(t), \ldots, u^{(m)}(t), g(t)\right)$

where,

$f\left(t, u(t), u^{\prime}(t), u^{\prime \prime}(t), \ldots, u^{(m)}(t), g(t)\right)=a_{m} u^{(m)}(t)+a_{m-1} u^{(m-1)}(t)+\ldots+$ $a_{1} u^{\prime}(t)+a_{0} u(t)+N\left(u(t), u^{\prime}(t)\right)-g(t)$ and the k-th residual function, $\operatorname{Res}_{\mathrm{k}}$, as follows:

$\operatorname{Re} s_{k}(t)=D_{t_{0}}^{a} u_{k}(t)-f\left(t, u_{k}(t)\right.$,
$\left.u_{k}^{\prime}(t), u_{k}^{\prime \prime}(t), \ldots, u_{k}^{(m)}(t), g(t)\right)$

It is clear that $\operatorname{Res}(t)=0$ and $\lim _{k \rightarrow \infty} \operatorname{Re} s_{k}(t)=\operatorname{Res}(t)$ for each $t \in\left(t_{0}, t_{0}+R\right)$. In fact, this lead to $D_{t_{0}}^{j a} \operatorname{Res}(t)=0$, since the fractional derivative of a constant function in the Caputo's sense is zero. In the mean time, the fractional derivatives $D_{t}^{i a}$ of $\operatorname{Res}(t)$ and $\operatorname{Res}_{k}(t)$ are matching at $t=t_{0}$ for each $i=0,1,2, \ldots, k$; that is, $D_{t_{0}}^{i a} \operatorname{Res}\left(t_{0}\right)=D_{t_{0}}^{i a} \operatorname{Res}_{k}\left(t_{0}\right)=0, i=0,1,2, \ldots, k$.

To obtain the rules form of coefficients $c_{j}, j=$ $1,2, \ldots, k$, in Equation (20), we apply the following 
subroutine: Substitute k-th truncated series of $\mathrm{u}(\mathrm{t})$ into Equation (23), find the fractional derivative formula $D_{t}^{(k-1) a}$ of $\operatorname{Res}_{\mathrm{k}}, \mathrm{k}=1,2,3, \ldots$ at $\mathrm{t}=\mathrm{t}_{0}$ and then finally solve the obtained algebraic equation to get the required coefficients. Anyhow, we need to solve the following algebraic equation:

$D_{t_{0}}^{(k-1) a} \operatorname{Res}_{k}\left(t_{0}\right)=0, m-1 \leq a \leq m, k=1,2,3, \ldots$

On the other aspect as well, to determine the form of the first unknown coefficient, $c_{1}$, we should substitute the 1-st truncated series, $u_{1}(t)$, of Equation (16) and (17) into the 1-st residual function, $\operatorname{Res}_{1}(t)$, of Equation (22) to get that:

$\operatorname{Res}_{1}(t)=D_{t_{0}}^{a} u_{1}(t)-f\left(t, u_{1}(t), u_{1}^{\prime}(t), u_{1}^{n_{1}}(t), \ldots, u_{1}^{(m)}(t), g(t)\right)$

But since $u_{1}(t)=b_{0}+c_{1} \frac{\left(t-t_{0}\right)^{a}}{\Gamma(a+1)}$, then Equation

lead to the following result:

$\operatorname{Res}_{1}(t)=c_{1}+f\left(\begin{array}{l}t, b_{0}+c_{1} \frac{\left(t-t_{0}\right)^{a}}{\Gamma(a+1)^{\prime}} \frac{d}{d t} \\ \left(\frac{c_{1}\left(t-t_{0}\right)^{a}}{\Gamma(a+1)}\right), . ., \frac{d^{m}}{d t^{m}}\left(\frac{c_{1}\left(t-t_{0}\right)^{a}}{\Gamma(a+1)}\right), g(t)\end{array}\right)$

Now, depending on the result of Equation (23) for $\mathrm{k}$ $=1$, the substitution of $\mathrm{t}=\mathrm{t}_{0}$ through Equation (25) yields that $\mathrm{c}_{1}=f\left(\mathrm{t}_{0}, \mathrm{~b}_{0}, 0,0, \ldots, 0, \mathrm{~g}\left(\mathrm{t}_{0}\right)\right)$. Hence, the 1 -st RPS approximate solution of Equation (16) and (17) can be expressed in the form of $u_{1}(t)=b_{0}+\frac{f\left(t_{0}, b_{0}\right)}{\Gamma(a+1)}\left(t-t_{0}\right)^{a}$ such that $f\left(t_{0}, b_{0}\right)=f\left(t_{0}, b_{0}, 0,0, \ldots, 0, g\left(t_{0}\right)\right)$.

Similarly, to find out the second unknown coefficient, $c_{2}$, we substitute the 2-nd truncated series, $u_{2}(t)=b_{0}+f\left(t_{0}, b_{0}\right) \frac{\left(t-t_{0}\right)^{a}}{\Gamma(a+1)}+c_{2} \frac{\left(t-t_{0}\right)^{2 a}}{\Gamma(2 a+1)} \quad$ into the 2nd residual

function, $\operatorname{Res}_{2}(t)=D_{t_{0}}^{a} u_{2}(t)-f\left(t, u_{2}(t), u_{2}^{\prime}(t), u_{2}{ }_{2}(t), \ldots, u_{2}^{(m)}(t), g(t)\right)$, to obtain the following result $\operatorname{Res}_{2}(t)=\left(f\left(t_{0}, b_{0}\right)+c_{2} \frac{\left(t-t_{0}\right)^{a}}{\Gamma(a+1)}\right):$

$$
-f\left(\begin{array}{l}
t,\left(b_{0}+f\left(t_{0}, b_{0}\right) \frac{\left(t-t_{0}\right)^{a}}{\Gamma(a+1)}+c_{2} \frac{\left(t-t_{0}\right)^{2 a}}{\Gamma(2 a+1)}\right), \frac{d}{d t} \\
\left(f\left(t_{0}, b_{0}\right) \frac{\left(t-t_{0}\right)^{a}}{\Gamma(a+1)}+c_{2} \frac{\left(t-t_{0}\right)^{2 a}}{\Gamma(2 a+1)}\right), . ., \frac{d^{m}}{d t^{m}} \\
\left(f\left(t_{0}, b_{0}\right) \frac{\left(t-t_{0}\right)^{a}}{\Gamma(a+1)}+c_{2} \frac{\left(t-t_{0}\right)^{2 a}}{\Gamma(2 a+1)}\right), g(t)
\end{array}\right)
$$

Now, applying the operator $D_{t_{0}}^{a}$ on both sides of Equation (26) and depending on the result $D_{t_{0}}^{(k-1) a} \operatorname{Res}_{k}\left(t_{0}\right)=0$ for $k=2$, then the value of $c_{2}$ will be given. This procedure can be repeated till the arbitrary order coefficients of the FPS solution for Equation (16) are obtained. Moreover, higher accuracy can be achieved by evaluating more components of the solution.

\section{Application to Time Fractional Wave-Like Differential Equation}

To demonstrate the simplicity and effectiveness of the proposed method, semi-analytical solutions for a class of inhomogeneous time-fractional equations is constructed using the FPS approach. The method is applied in a direct way without using linearization, transformation, or restrictive assumptions. The results reveal that the method is highly accurate, rapidly converge and convenient to handle a various engineering problems in fractional calculus. Consider the following time fractional wave-like partial differential equation:

$$
\begin{aligned}
& D_{t}^{a} u=x^{2}+y^{2}+z^{2}+\frac{1}{2}\left(x^{2} u_{x x}+y^{2} u_{y y}+z^{2} u_{z z}\right), \\
& 0<x, y, z<1,1<a \leq 2, t>0
\end{aligned}
$$

Subject to the boundary conditions:

$$
\begin{aligned}
& u(0, y, z, t)=y^{2}\left(e^{t}-1\right)+z^{2}\left(e^{-t}-1\right), \\
& u(1, y, z, t)=\left(1+y^{2}\right) f(t)+z^{2}\left(e^{-t}-1\right), \\
& u(x, 0, z, t)=x^{2}\left(e^{t}-1\right)+z^{2}\left(e^{-t}-1\right), \\
& u(x, 1, z, t)=\left(1+x^{2}\right) f(t)+z^{2}\left(e^{-t}-1\right), \\
& u(x, y, 0, t)=\left(x^{2}+y^{2}\right)\left(e^{t}-1\right) \\
& u(x, y, 1, t)=\left(x^{2}+y^{2}\right)\left(e^{t}-1\right)+\left(e^{-t}-1\right)
\end{aligned}
$$

And the initial conditions:

$$
u(x, y, z, 0)=0, \quad u_{t}(x, y, z, 0)=x^{2}+y^{2}-z^{2}
$$

where, $D_{t}^{a}$ is Caputo fractional derivative of order a with respect to the independent variable $t$.

From the conditions (28) and (29), one can get the following transformed form:

$u(x, y, z, t)=\left(x^{2}+y^{2}\right) f(t)+z^{2} g(t)$

Substituting Equation (30) into Equation (27), we have:

$$
\begin{aligned}
& \left(x^{2}+y^{2}\right) D_{t}^{a} f(t)+z^{2} D_{t}^{a} g(t) \\
& =\left(x^{2}+y^{2}\right)(1+f(t))+z^{2}(1+g(t))
\end{aligned}
$$

Which yields two time fractional ordinary differential equations: 


$$
\begin{array}{ll}
D_{t}^{a} f(t)=1+f(t), & 1<a \leq 2, t<0 \\
D_{t}^{a} g(t)=1+g(t), & 1<a \leq 2, t>0
\end{array}
$$

Subject to the initial conditions:

$f(0)=0, f^{\prime}(0)=1, g(0)=0, g^{\prime}(0)=-1$

In order to apply the RPS method to the system of fractional differential Equations (32) and (33), we assume that the functions $f(t)$ and $g(t)$ can be expanded by FPS representation in Theorem 3. As follows:

$$
f(t)=\sum_{i=0}^{\infty} \sum_{j=0}^{1} a_{i j} t^{j+i a}, g(t)=\sum_{i=0}^{\infty} \sum_{j=0}^{1} b_{i j} t^{j+i a}
$$

where, $a_{i j}$ and $b_{i j}$ are coefficients to be determined.

From the initial conditions (33), we can conclude that $a_{00}=0, a_{01}=1, b_{00}=0$ and $b_{01}=-1$. So, the series (34) can be written as:

$$
\begin{aligned}
& f(t)=t+\sum_{i=1}^{\infty} \sum_{j=0}^{1} a_{i j} t^{j+i a} \\
& g(t)=-t+\sum_{i=1}^{\infty} \sum_{j=0}^{1} b_{i j} t^{j+i a}
\end{aligned}
$$

Thus the approximate solutions of Equation (32) and (33) can be represented by the $(k, 1)$-truncated series:

$$
\begin{aligned}
& f_{k, l}(t)=t+\sum_{i=1}^{k} \sum_{j=0}^{l} a_{i j} t^{j+i a}, \\
& g_{k, l}(t)=-t+\sum_{i=1}^{k} \sum_{j=0}^{l} b_{i j} t^{j+i a}
\end{aligned}
$$

Prior to applying the RPS technique for finding the values of coefficients $a_{i j}$ and $b_{i j}$ in the series expansions (36), we must define the residual functions concept for the main system of fractional differential equation (32) as

$$
\operatorname{Res}^{1}(t)=D_{t}^{a} f(t)-f(t)-1,
$$

$t \geq 0, \operatorname{Res}^{2}(t)=D_{t}^{a} g(t)-g(t)-1, t \geq 0, \quad$ whereas the truncated $(\mathrm{k}, 1)$-resudial functions are defined as follows:

$$
\begin{aligned}
& \operatorname{Res}_{(k, \mathrm{l})}^{1}(t)=D_{t}^{a} f_{k, l}(t)-f_{k, l}(t)-1, t \geq 0 \\
& \operatorname{Res}_{(k, 1)}^{2}(t)=D_{t}^{a} g_{k, l}(t)-g_{k, l}(t)-1, t \geq 0
\end{aligned}
$$

As we mentioned earlier, it is clear that $\operatorname{Res}^{n}(t)=0$ for each $t \in\left[0, \mathrm{R}_{\mathrm{n}}\right), n=1,2$, where $R_{n}, n=1,2$ is the radius of convergence of series (14) and (15) respectively. In fact, this shows that $D_{t}^{(i-1) a} D_{t}^{j} \operatorname{Res}(t)=0$ for each $i=1,2,3, \ldots, k$ and $j=0,1$, since the fractional derivative of a constant function in the Caputo sense is zero. In the meantime, the fractional derivatives
$D_{t}^{(i-1) a} D_{t}^{j}$ for each $\mathrm{i}=1,2,3, \ldots, \mathrm{k}$ and $\mathrm{j}=0,1$ of $\operatorname{Res}^{\mathrm{n}}(\mathrm{t})$ and $\operatorname{Res}_{(k, l)}^{n}(t), n=1,2$ are matching at $\mathrm{t}=0$, it is obvious that:

$D_{t}^{(i-1) a} D_{t}^{j} \operatorname{Re} s^{n}(0)=D_{t}^{(i-1) a} \operatorname{Res}_{(k, l)}^{n}(0)$

$=0, i=1,2,3, \ldots, k, j=0,1, n=1,2$

To obtain the value of coefficients $a_{10}$ and $b_{10}$ in Equation (35), we find out the $(1,0)$-truncated series of the solutions of system (32) as follows:

$$
\begin{aligned}
& f_{1,0}(t)=t+a_{10} t^{a}, \\
& g_{1,0}(t)=-t+b_{10} t^{a}
\end{aligned}
$$

Substitute Equation (39) back into both sides of the residual functions, $\operatorname{Res}_{(1,0)}^{n}, n=1,2$ respectively, to get the following result:

$$
\begin{aligned}
& \operatorname{Res}_{(1,0)}^{1}(t)=a_{10} \Gamma(a+1)-t-a_{10} t^{a}-1, \\
& \operatorname{Res}_{(1,0)}^{2}(t)=b_{10} \Gamma(a+1)+t-b_{10} t^{a}-1
\end{aligned}
$$

Now, depending on the result of Equation (38) for $(i, j)$ $=(1,0)$, we have $a_{10}=b_{10}=\frac{1}{\Gamma(a+1)}$. Hence, the $(1,0)$-RPS approximation of system (32) can be expressed as follows:

$$
\begin{aligned}
& f_{1,0}(t)=t+\frac{1}{\Gamma(a+1)} t^{a}, \\
& g_{1,0}(t)=-t+\frac{1}{\Gamma(a+1)} t^{a}
\end{aligned}
$$

Again, to find out the value of the unknown coefficient, $a_{11}$ and $b_{11}$ in Equation (36), we must find and formulate the $(1,1)$-truncated series expansion for system (32) as follows:

$$
\begin{aligned}
& f_{1,1}(t)=t+\frac{1}{\Gamma(a+1)} t^{a}+a_{11} t^{1+a} \\
& g_{1,1}(t)=-t+\frac{1}{\Gamma(a+1)} t^{a}+a_{11} t^{1+a}
\end{aligned}
$$

And the (1,1)-residual functions based on Equation (38) as follows:

$$
\begin{aligned}
& \operatorname{Res}_{1,1}^{1}(t)=a_{11} \Gamma(a+2) t-t-\frac{1}{\Gamma(a+1)} t^{a}-a_{11} t^{1+a} \\
& \operatorname{Res}_{1,1}^{2}(t)=b_{11} \Gamma(a+2) t+t-\frac{1}{\Gamma(a+1)} t^{a}-b_{11} t^{1+a}
\end{aligned}
$$

By applying the operator $D_{t}$ to the both side of Equation (43), we get that: 


$$
\begin{aligned}
& D_{t} \operatorname{Res}_{(1,1)}^{1}(t)=a_{11} \Gamma(a+2)-1-\frac{1}{\Gamma(a)} t^{a-1}-a_{11}(1+a) t^{a} \\
& D_{t} \operatorname{Res}_{(1,1)}^{2}(t)=b_{11} \Gamma(a+2)+1-\frac{1}{\Gamma(a)} t^{a-1}-b_{11}(1+a) t^{a}
\end{aligned}
$$

Using the fact in Equation (38), that is, $D_{t} \operatorname{Res}_{(1,1)}^{n}(0)=0, n=1,2$, we can easily obtain $a_{11}=-b_{11}=\frac{1}{\Gamma(a+2)}$. Hence, the $(1,1)$-RPS approximation for system (32) can be expressed as:

$$
\begin{aligned}
& f_{1,1}(t)=t+\frac{1}{\Gamma(a+1)} t^{a}+\frac{1}{\Gamma(a+2)} t^{1+a} \\
& g_{1,1}(t)=-t+\frac{1}{\Gamma(a+1)} t^{a}-\frac{1}{\Gamma(a+2)} t^{1+a}
\end{aligned}
$$

In general, to determine the value of coefficients $\mathrm{a}_{\mathrm{vw}}$ and $b_{\mathrm{vw}}$ in Equation (35) for $\mathrm{v}=1,2,3, \ldots$ and $\mathrm{w}=0,1$, we apply the following subroutine: substitute $(\mathrm{v}, \mathrm{w})-$ truncated series approximation of $f(\mathrm{t})$ and $\mathrm{g}(\mathrm{t})$ into Equatiom (37), find out the fractional derivative formulas $D_{t}^{(v-1) a} D_{t}^{w}$ of $\operatorname{Res}_{(v, w)}^{n}(t)$ at $\mathrm{t}=0$ and then finally solve the obtained algebraic equation (38) to get the required coefficients. Therefore, if we apply the previous generality, then we obtain that:

$$
\begin{aligned}
& a_{v 0}=b_{v 0}=\frac{1}{\Gamma(1+v a)} t^{v a}, \\
& a_{v 1}=-b_{v 1}=\frac{1}{\Gamma(2+v a)} t^{1+v a}
\end{aligned}
$$

Thus, the FPS expansions of the solutions for system (32) are given by:

$$
\begin{aligned}
& f(t)=t+\sum_{i=1}^{\infty} \sum_{j=1}^{1} \frac{1}{\Gamma(j+1+i a)} t^{j+i a} \\
& =\sum_{i=1}^{\infty} \frac{t^{i a}}{\Gamma(1+i a)}+t \sum_{i=0}^{\infty} \frac{t^{i a}}{\Gamma(2+i a)}=E_{a}\left(t^{a}\right)+t E_{a, 2}\left(t^{a}\right) \\
& g(t)=-t+\sum_{i=1}^{\infty} \sum_{j=0}^{1} \frac{(-1)^{j}}{\Gamma(j+1+i a)} t^{j+i a} \\
& =\sum_{i=1}^{\infty} \frac{t^{i a}}{\Gamma(1+i a)}+t \sum_{i=0}^{\infty} \frac{t^{i a}}{\Gamma(2+i a)}=E_{a}\left(t^{a}\right)-t E_{a, 2}\left(t^{a}\right)
\end{aligned}
$$

where, $E_{a}\left(t^{a}\right)$ and $E_{a, 2}\left(t^{a}\right)$ are the Mittag-Leffler function and the generalized Mittag-Leffler function, respectively.

Therefore, the solution of the time fractional wavelike partial differential equation (27) will be in the following form:

$$
u(x, y, z, t)=\left(x^{2}+y^{2}+z^{2}\right) E_{a}\left(t^{a}\right)+\left(x^{2}+y^{2}-z^{2}\right) t E_{a, 2}\left(t^{a}\right)
$$

Which is exactly the same as those given by the Adomian decomposition method and Homotopy analysis method. Especially, when $a=2$, the solution of Equation (27) is given by:

$$
u(x, y, z, t)=\left(x^{2}+y^{2}+z^{2}\right) E_{2}\left(t^{a}\right)+\left(x^{2}+y^{2}-z^{2}\right) t E_{2,2}\left(t^{2}\right)
$$

\section{Conclusion}

The main concern of this work has been to propose an efficient method for the solution of fractional differential equations with time fractional derivatives. The goal has been achieved by extending the PSM to solve this class of FDEs. We can conclude that the fractional PSM is powerful and efficient technique in finding approximate solutions for linear and nonlinear FIVPs. The proposed algorithm produced a rapidly convergent series under a multiple fractional differentiability with easily computable components. After computing several approximations and using the boundary conditions at the boundary points, we can easily determine the approximate solution. This confirms our belief that the efficiency of our technique gives it much wider applicability for general classes of linear and nonlinear problems.

\section{Acknowledgment}

The authors are grateful to the referees for their helpful suggestions that improved this article.

\section{Author's Contributions}

Radwan Abu-Gdairi: Designed the research plan, organized the study and contributed to the writing of the manuscript.

Mohammed Al-Smadi: Contributed to the writing and revising of the manuscript, as well as coordinated the mouse work.

Ghaleb Gumah: Participated in all experiments, coordinated the data-analysis and contributed to the writing of the manuscript.

\section{Funding Information}

This research is funded by the Deanship of Research in Zarqa University/Jordan.

\section{Ethics}

This article is original and contains unpublished material. The corresponding author confirms that all of the other authors have read and approved the manuscript and no ethical issues involved.

\section{Conflict of Interests}

The authors declare that there is no conflict of interests regarding the publication of this paper. 


\section{References}

Abbasbandy, S., 2007. An approximation solution of a nonlinear equation with Riemann-Liouville's fractional derivatives by He's variational iteration method. J. Computational Applied Math., 207: 53-58. DOI: 10.1016/j.cam.2006.07.011

Al-Smadi, M., 2013. Solving initial value problems by residual power series method. Theoretical Math. Applications, 3: 199-210.

Al-Smadi, M., O. Abu Arqub and S. Momani, 2013. A Computational method for two-point boundary value problems of fourth-order mixed integrodifferential Equations. Math. Problems Eng. DOI: $10.1155 / 2013 / 832074$

Arıkoğlu, A. and I. Özkol, 2007. Solution of fractional differential equations by using differential transform method. Chaos Solitons Fractals, 34: 1473-1481. DOI: $10.1016 /$ j.chaos.2006.09.004

Arqub, A.O., 2013. Series solution of fuzzy differential equations under strongly generalized differentiability. J. Advanced Res. Applied Math., 5: 31-52. DOI: 10.5373/jaram.1447.051912

Arqub, A.O., M. Al-Smadi and N. Shawagfeh, 2013. Solving Fredholm integro-differential equations using reproducing kernel Hilbert space method. Applied Math. Computation, 219: 8938-8948. DOI: $10.1016 /$ j.amc.2013.03.006

Arqub, A.O., M. Al-Smadi and S. Momani, 2012. Application of reproducing kernel method for solving nonlinear Fredholm-Volterra integrodifferential equations. Abstract Applied Analysis. DOI: $10.1155 / 2012 / 839836$

Arqub, A.O., M. Al-Smadi, S. Momani and T. Hayat, 2015. Numerical solutions of fuzzy differential equations using reproducing kernel hilbert space method. Soft Computing. DOI: 10.1007/s00500-015-1707-4

Arqup, O.A. and A. El-Ajou, 2013. Solution of the fractional epidemic model by homotopy analysis method. J. King Saud University Sci., 25: 73-81. DOI: $10.1016 /$ j.jksus.2012.01.003

Caputo, M., 1967. Linear models of dissipation whose Q is almost frequency independent. Part II. J. Roy Austral Soc., 13: 529-539.

El-Ajou, A., O.A. Arqub and M. Al-Smadi, 2015. A general form of the generalized Taylor's formula with some applications. Applied Math. Computation, 256: 851-859.

DOI: $10.1016 /$ j.amc.2015.01.034

El-Ajou, A., Z. Odibat, S. Momani and A. Alawneh, 2010. Construction of analytical solutions to fractional differential equations using homotopy analysis method. Int. J. Applied Math., 40: 43-43.

Ertürk, V.S. and S. Momani, 2008. Solving systems of fractional differential equations using differential transform method. J. Computational Applied Math., 215: 142-151. DOI: 10.1016/j.cam.2007.03.029
Hashim, I., O. Abdulaziz and S. Momani, 2009. Homotopy analysis method for fractional IVPs. Commun. Nonlinear Sci. Numerical Simulation, 14: 674-684. DOI: 10.1016/j.cnsns.2007.09.014

He, J.H., 1999. Some applications of nonlinear fractional differential equations and their approximations. Bull. Sci. Technol., 15: 86-90.

Komashynska, I., M. Al-Smadi, O. Abu Arqub, S. Momani, 2015. An efficient analytical method for solving singular initial value problems of nonlinear systems. Applied Math. Inf. Sci.

Luchko, Y. and R. Gorenflo, 1998. The Initial Value Problem for Some Fractional Differential Equations with the Caputo Derivatives. 1st Edn., Fachbereich Mathematik und Informatic, Berlin, pp: 23.

Mainardi, F., 1997. Fractional Calculus: Some Basic Problems in Continuum and Statistical Mechanics. In: Fractals and Fractional Calculus in Continuum Mechanics, A. Carpenter and F. Mainardi, (Eds.), Springer-Verlag, New York, pp: 291-348.

Miller, K.S. and B. Ross, 1993. An Introduction to the Fractional Calculus and Fractional Differential Equations. 1st Edn., Wiley, New York, ISBN-10: $0471588849,384$.

Moaddy, K., M. AL-Smadi and I. Hashim, 2015. A novel representation of the exact solution for differential algebraic equations system using residual power-series method. Discrete Dynamics Nature Society Article, 2015: 12-12. DOI: 10.1155/2015/205207

Molliq, Y..R, M.S.M. Noorani and I. Hashim, 2009. Variational iteration method for fractional heat- and wave-like equations. Nonlinear Analysis Real World Applications, 10: 1854-1869.

DOI: 10.1016/j.nonrwa.2008.02.026

Momani, S. and N.T. Shawagfeh, 2002. Decomposition method for solving fractional Riccati differential equations. Appl. Math. Comput., 182: 1083-1092. DOI: $10.1016 /$ j.amc.2006.05.008

Momani, S. and Z. Odibat, 2007. Homotopy perturbation method for nonlinear partial differential equations of fractional order. Phy. Lett. A, 365: 345-350. DOI: 10.1016/j.physleta.2007.01.046

Momani, S. and Z. Odibat, 2007. Numerical comparison of methods for solving linear differential equations of fractional order. Chaos, Solitons Fractals, 31: 1248-1255. DOI: 10.1016/j.chaos.2005.10.068

Momani, S., A. Freihat and M. AL-Smadi, 2014. Analytical study of fractional-order multiple chaotic FitzHugh-Nagumo neurons model using multistep generalized differential transform method. Abstract Applied Analysis. DOI: 10.1155/2014/276279

Odibat, Z. and S. Momani, 2006. Application of variation iteration method to nonlinear differential equations of fractional order. Int. J. Nonlin. Sci. Numer. Simulat., 1: 15-27. DOI: 10.1515/IJNSNS.2006.7.1.27 
Odibat, Z. and S. Momani, 2008. Modified homotopy perturbation method: Application to quadratic riccati differential equation of fractional order. Chaos, Solitons Fractals, 36: 167-174.

DOI: $10.1016 /$ j.chaos.2006.06.041

Odibat, Z.M. and N.T. Shawagfeh, 2007. Generalized taylor's formula. Appl. Math. Comput., 186: 286-293. DOI: 10.1016/j.amc.2006.07.102

Oldham, K.B. and J. Spanier, 1974. The Fractional Calculus. 1st Edn., Academic Press, New York.

Oturanç, G., A. Kurnaz and Y. Keskin, 2008. A new analytical approximate method for the solution of fractional differential equations. Int. J. Comput. Math., 85: 131-142. DOI: 10.1080/00207160701405477

Podlubny, I., 1999. Fractional Differential Equations. 1st Edn., Academic Press, New York.
Schneider, W.R., 1996. Completely monotone generalized mittag-leffler functions. Expo. Math., 14: 3-16.

Shawagfeh, N.T., 1999. The decomposition method for fractional differential equations. J. Frac. Calc., 16: 27-33.

Shawagfeh, N.T., 2002. Analytical approximate solutions for nonlinear fractional differential equations. Appl. Math. Comput., 131: 517-529. DOI: $10.1016 /$ S0096-3003(01)00167-9

Song, L. and H. Zhang, 2007. Application of homotopy analysis method to fractional KdV-BurgersKuramoto equation. Phy. Lett. A, 367: 88-94. DOI: $10.1016 /$ j.physleta.2007.02.083

Young, G.O., 1995. Definition of physical consistent damping laws with fractional derivatives. Z. Angew. Math. Mech., 75: 623-35. 